\title{
Disentangling excited states
}

\author{
Kimmy K. Cushman* \\ Yale University \\ E-mail: kimmy.cushman@yale.edu

\section{George T. Fleming} \\ Yale University \\ E-mail: george.fleming@yale.edu
}

\begin{abstract}
In lattice gauge theory, understanding contributions from excited states is imperative for achieving high precision calculations. A variety of methods are available to extract excited states, such as fitting to multiple exponentials, Prony's method, and Matrix Prony, correlator matrices, and generalized eigenvalue problems. A generic problem faced by all these methods is that the resulting states tend to have overlapping error ellipses (e.g. jackknife, bootstrap, cross-validation, etc.) making identification of states ambiguous. The problem may be alleviated somewhat by expert guidance in operator selection to minimize overlap for a few low-lying states, but this defeats the overall design goal of an automated black-box method. Instead, we face the overlapping states labeling problem directly. For example, using the bootstrapping method, resolving excited state energies and their error bars requires finding the most probable set of state labels for each bootstrap sample. We investigate several variants of expectation maximization clustering in attempt to find an efficient algorithm for bootstrap labeling and therefore state identification.
\end{abstract}

The 37th Annual International Symposium on Lattice Field Theory - LATTICE2019

16-22 June, 2019

Hilton Hotel Wuhan Riverside, Wuhan, China.

\footnotetext{
* Speaker.
} 
In this work, we investigated the labeling problem which occurs when using an algebraic method of extracting excited states from lattice correlation functions. The algebraic method discussed here is Prony's method, derived from the original work $[1,2]$ for use in lattice correlation functions [3-7]. In our presentation, we mainly address the problem using clustering algorithms, following the work in [8], but we find this approach to be insufficient. Recently, we have found a suitable solution to the labeling problem which we call automated label flows, based on an idea originating in [9]. The shortcomings of our original approach and a discussion of the automated label flows solution and how it overcomes the labeling problem is presented in [10], which can be found at https://arxiv.org/abs/1912.08205.

\section{References}

[1] G. C. F. M. R. de Prony. J. Ecole Poly, 1:24, 1795.

[2] David Vandevoorde. A fast exponential decomposition algorithm and its applications to structured matrices. PhD thesis, Rensselaer Polytechnic Institute, Troy, New York, USA, ProQuest Dissertations Publishing, 1996.

[3] George Tamminga Fleming. What can lattice QCD theorists learn from NMR spectroscopists? In QCD and numerical analysis III. Proceedings, 3rd International Workshop, Edinburgh, UK, June 30-July 4, 2003, pages 143-152, 2004.

[4] George T. Fleming, Saul D. Cohen, Huey-Wen Lin, and Victor Pereyra. Excited-State Effective Masses in Lattice QCD. Phys. Rev., D80:074506, 2009.

[5] Huey-Wen Lin Saul Cohen, George Fleming. Exponential time series in lattice quantum field theory. In V. Pereyra and G. Scherer, editors, Exponential Data Fitting and Its Applications, chapter 4, pages 71-93. Bentham eBooks, 2010.

[6] Evan Berkowitz, Amy Nicholson, Chia Cheng Chang, Enrico Rinaldi, M. A. Clark, Bálint Joó, Thorsten Kurth, Pavlos Vranas, and André Walker-Loud. Calm Multi-Baryon Operators. EPJ Web Conf., 175:05029, 2018.

[7] S. Romiti and S. Simula. Extraction of multiple exponential signals from lattice correlation functions. Phys. Rev., D100(5):054515, 2019.

[8] Kimmy K. Cushman and George T. Fleming. Prony methods for extracting excited states. PoS, LATTICE2018:297, 2019.

[9] Daniel Hoying. Improved Pion Correlation Functions Under Lattice Action. PhD thesis, University of Connecticut, Storrs, Connecticut, USA, 2019.

[10] Kimmy K. Cushman and George T. Fleming. Automated label flows for excited states of correlation functions in lattice gauge theory. 2019. 